\title{
Lipid-lowering therapy in high cardiovascular risk patients during COVID-19 pandemic: keep focused on the target
}

\author{
Arturo Cesaro" ${ }^{1,2}$, Carmine Riccioº ${ }^{2}$ Paolo Calabrò ${ }^{1,2}$ \\ ${ }^{1}$ Department of Translational Medical Sciences, University of Campania "Luigi Vanvitelli", Naples; ${ }^{2}$ Division of \\ Clinical Cardiology, A.O.R.N. "Sant'Anna e San Sebastiano", Caserta, Italy
}

To the Editor

COVID-19 (COrona VIrus Disease) patients with cardiovascular $(\mathrm{CV})$ disease, multiple $\mathrm{CV}$ risk factors or comorbidities (i.e., arterial hypertension and diabetes) were shown to be more prone to worse prognosis [1]. SARS-CoV-2 is a still unknown enemy and the role of concomitant cardiovascular therapies has been controversial in the early stages, particularly with regard to Angiotensin-Converting Enzyme inhibitors.

The aims of this letter are: i) to assess the appropriateness to continue lipid-lowering therapy (LLT) in COVID-19 patients, ii) to discuss the strategies to avoid undertreatment in non-COVID patients at high $\mathrm{CV}$ risk during pandemic.

LLT is a cornerstone in the treatment of high CV risk patients and statins are the most widely used drugs. To date, there is no evidence to justify discontinuation of LLT in COVID-19 patients. On the other hand, several studies have shown that patients with viral infections or pneumonia treated with statins had a reduction in $\mathrm{CV}$ outcomes and mortality rate [2-4]. A large cohort study highlights a reduction in death rate in both chronic obstructive pulmonary dis-

Correspondence: Prof. Paolo Calabrò, Division of Clinical Cardiology, A.O.R.N. Sant'Anna e San Sebastiano, Caserta; Department of Translational Medical Sciences, University of Campania "Luigi Vanvitelli", 80131 Naples, Italy.

Tel. +39.0823.232395 - Fax: +39.0823.232395

E-mail: paolo.calabro@unicampania.it

Contributions: All the authors made a substantive intellectual contribution, have read and approved the final version of the manuscript and agreed to be accountable for all aspects of the work.

Conflict of interest: The authors declare that they have no competing interests, and all authors confirm accuracy.

Key words: COVID-19; lipid lowering therapy; statin; PCSK9; Coronavirus; cardiac rehabilitation; dyslipidemia.

Received for publication: 5 December 2020 .

Accepted for publication: 8 January 2021

${ }^{\circ}$ Copyright: the Author(s), 2021

Licensee PAGEPress, Italy

Monaldi Archives for Chest Disease 2021; 91:1721

doi: 10.4081/monaldi.2021.1721

This article is distributed under the terms of the Creative Commons Attribution Noncommercial License (by-nc 4.0) which permits any noncommercial use, distribution, and reproduction in any medium, provided the original author(s) and source are credited. ease and influenza patients on statins treatment [3]. The intense inflammatory response and hemodynamic modifications in patients with severe symptoms of COVID, could contribute to the atherosclerotic plaques rupture resulting in acute ischemic event. In this scenario, LLT, as in routine clinical practice, could contribute to plaques stabilization and avoid acute ischemic injuries which are predictors of mortality in high-risk COVID-19 patients [1].

Among the controversial statins' pleiotropic effects, they seem to modulate the immune response, immune cell adherence and cytokine secretion. In fact, besides reducing cholesterol biosynthesis by inhibition of 3-hydroxy-3-methylglutaryl-coenzyme-A reductase, statins interfere with the mevalonate pathway preventing the synthesis of intermediate isoprenoid products such as farnesyl-pyrophosphate and geranyl-geranyl-pyrophosphate.

There are clinical and laboratory evidence showing an interference of the statins with viral infections. In that regard, recently, in vitro study showed that statin treatment seems to reduce infectious Ebola virus in primary human monocyte-derived macrophages and in the hepatic cell line Huh7 [5]. Moreover, statins are recognized inhibitors of the MYD88 pathway which is highly induced by SARS-CoV-1 with activation of the NF-kB pathway resulting in increased inflammation. The aptitude of statins to preserve MYD88 normal levels or its immunomodulatory action may be useful in COVID-19 patients, in whom an excessive inflammatory response causes acute respiratory distress syndrome [6]. Recent studies have shown an association between hypolipidemia and severity of COVID-19. Both total and lowdensity cholesterol were significantly lower during severe COVID-19 infection, but this should not suggest a cholesterol causal link in clinical worsening, but rather a consequence of the disease. Growing evidence seeks to shed light on statin use in these patients, and the results are not univocal. Recent data have shown that antecedent statin use in patients hospitalized with COVID-19 was associated with lower mortality [7-9], even in patients with diabetes mellitus [10], and lower risk of acute respiratory distress syndrome [8] or invasive mechanical ventilation [11]. Furthermore, patients with statin use prior to admission seem to have a lower risk of developing severe COVID-19, and a faster time to recovery [12]. Data on 4842 patients from Danish nationwide registries showed that prior statin therapy in patients with COVID-19 had a neutral impact on all-cause mortality or severe infection [13]. On the other hand, the CORONADO study, a French nationwide observational study, showed that routine statin treatment is associated with increased mortality in patients with diabetes hospitalized for COVID-19 [14].

A meta-analysis of four studies with a total of 8990 COVID19 patients showed a significantly reduced hazard $(\mathrm{HR}=0.70 ; 95 \%$ CI 0.53-0.94) for fatal or severe disease with the use of statins [15]; whereas 2 other meta-analyses showed a neutral effect of 
statins with no significant reductions in in-hospital mortality or COVID-19 severity [16,17]. The Intermediate versus Standarddose Prophylactic anticoagulation In cRitically-ill pATIents with COVID-19: An opeN label randomized controlled trial (INSPIRATION) and INSPIRATION-statin (INSPIRATION-S) studies will give us information about statins efficacy and safety in COVID-19 patients [18]. For all these reasons, albeit safety of statins in patients with COVID-19 has not yet been established, there is no evidence to suggest suspension, so it can be speculated that statins might have a positive effect. Of note, interactions and side effects should not be underestimated. Actually, protease inhibitor-based antiretrovirals, usually used in HIV-infection, are used in treatment of COVID-19 and treatment-related hyperlipidemia is already managed with statins in clinical practice. In patients treated with lopinavir/ritonavir, statins should be used with caution due to an increased risk of rhabdomyolysis. Furthermore, in patients with SARS-CoV-2 infection who experience liver impairment, statin administration should be conducted with caution and considering temporary interruption. There is no evidence about non-statin lipid lowering agents in COVID-19 patients. We can suppose that ezetimibe and PCSK9 inhibitors (PCSK9i) could be continued. In patient treated with PCSK9i the treatment may be continued with a detailed assessment of patients who experienced common adverse events like nasopharyngitis, upper respiratory tract infections and influenza. PCSK9 is involved in the inflammatory response, but its inhibition in inflammatory or septic clinical scenario is actually only an attractive hypothesis [19].

To date, the position statement from HEART UK [20] recommends not suspending LLT but to monitor for possible drug interactions, particularly in patients with impaired liver function.

The COVID-19 outbreak is impacting on inpatient/outpatient healthcare organization and the chronic patient management. It is relevant to point out the indirect effects of COVID-19 pandemic on the management of patients undergoing LLT. In order to contain the spread of SARS-CoV-2 contagion, a third of the global population has been on coronavirus lockdown or nationwide quarantine. During lockdown, data on hospital inflows in various regions of the world have shown a decrease in hospital admissions. In some countries, outpatient clinics have been suspended, but hospitals have guaranteed access to emergency medical care, also nonCOVID related. In this context, many patients have put aside the need for chronic pharmacological treatments.

For these reasons, the pandemic indirect effects on the CV risk should not be underestimated. In this journal, Mureddu et al. [21] have highlighted the relevance of secondary prevention programs during the pandemic, suggesting telemedicine networks. Also, the authors have emphasized the need to avoid suspension of cardiac rehabilitation and have proposed a new model of home-based cardiac rehabilitation exploiting social networking and digitalization. Against pandemic background, patients at high CV risk, for whom medical therapy is essential, risk having difficulty in accessing drugs and/or dose adjustments. Patients may avoid consulting physician for prescriptions, may stop life-saving therapies based on fake news or unproven theories that claim increased risk of viral infection, or have administrative difficulties in dispensing drugs. Patients on treatment with statins, ezetimibe, PCSK9i or fibrates should not stop taking their drugs. Some of mentioned lipid-lowering drugs require electronic prescriptions based on clinical and blood test data with scheduled deadlines. During the pandemic many patients did not have access to outpatient clinics and prescription renewal and they stopped treatments. New therapeutic strategies have been shown to increase therapeutic adherence and quality of life; discontinuation of these drugs could result in a loss of adherence and worse cardiovascular outcomes [22-24]. A chronic patient who discontinues treatment may never take the medication again. In this context, physicians should promote care of chronic patients by increasing remote monitoring systems and taking advantage of telehealth. Thanks to telehealth it is possible to activate a multidisciplinary network to monitor patients, assist them in chronic diseases and promote prevention. We can use this approach in dyslipidemic patients, contacting frail patients who have missed the scheduled visit and rescheduling them according to their clinical condition, monitoring biochemical parameters, evaluate drug prescription or dosage changes and organizing with pharmacies and pharmaceutical companies home drug delivery services. Although the pandemic puts a strain on the work of all physicians, implementing these actions could benefit the patient's psychological state and adherence to therapy, which are among the key determinants in the prevention of cardiovascular events.

In conclusion, the significant effect on $\mathrm{CV}$ prevention of LLT support its use and continuation in COVID-19 patients. Although there are some hypotheses suggesting beneficial statins action, further studies on concomitant medications in these patients are needed to provide more information on the outcomes. A new challenge in $\mathrm{CV}$ prevention and rehabilitation is ongoing; and despite the medical community rightly focusing on COVID-19 patients, we do not forget chronic patients, we need to keep focus on the target.

\section{References}

1. Wu Z, McGoogan JM. Characteristics of and important lessons from the coronavirus disease 2019 (COVID-19) outbreak in China: Summary of a report of 72314 cases from the Chinese Center for Disease Control and Prevention. JAMA 2020;323:1239-42.

2. Fedson DS. Treating influenza with statins and other immunomodulatory agents. Antiviral Res 2013;99:417-35.

3. Frost FJ, Petersen H, Tollestrup K, Skipper B. Influenza and COPD mortality protection as pleiotropic, dose-dependent effects of statins. Chest 2007;131:1006-12.

4. Douglas I, Evans S, Smeeth L. Effect of statin treatment on short term mortality after pneumonia episode: cohort study. BMJ 2011;342:d1642.

5. Shrivastava-Ranjan P, Flint M, Bergeron É, et al. Statins suppress Ebola virus infectivity by interfering with glycoprotein processing. mBio 2018;9:e00660-18.

6. Yuan S. Statins may decrease the fatality rate of middle east respiratory syndrome infection. mBio 2015;6:e 01120.

7. Gupta A, Madhavan M V, Poterucha TJ, et al. Association between antecedent statin use and decreased mortality in hospitalized patients with COVID-19. Res Sq 2020; rs.3.rs-56210. Preprint.

8. Fan Y, Guo T, Yan F, et al. Association of statin use with the inhospital outcomes of 2019-coronavirus disease patients: A retrospective study. Front Med (Lausanne) 2020;7:584870.

9. Zhang X-J, Qin J-J, Cheng X, et al. In-hospital use of statins is associated with a reduced risk of mortality among individuals with COVID-19. Cell Metab 2020;32:176-87.e4.

10. Saeed O, Castagna F, Agalliu I, et al. Statin use and in-hospital mortality in diabetics with COVID-19. J Am Heart Assoc 2020;9:e018475.

11. Song SL, Hays SB, Panton CE, et al. Statin use is associated with decreased risk of invasive mechanical ventilation in 
COVID-19 patients: A preliminary study. Pathogens 2020;9:759.

12. Daniels LB, Sitapati AM, Zhang J, et al. Relation of statin use prior to admission to severity and recovery among COVID-19 inpatients. Am J Cardiol 2020;136:149-55.

13. Butt JH, Gerds TA, Schou M, et al. Association between statin use and outcomes in patients with coronavirus disease 2019 (COVID-19): a nationwide cohort study. BMJ Open 2020;10:e044421.

14. Cariou B, Goronflot T, Rimbert A, et al. Routine use of statins and increased COVID-19 related mortality in inpatients with type 2 diabetes: Results from the CORONADO study. Diabetes Metab 202;101202.

15. Kow CS, Hasan SS. Meta-analysis of effect of statins in patients with COVID-19. Am J Cardiol 2020;134:153-5.

16. Hariyanto TI, Kurniawan A. Statin therapy did not improve the in-hospital outcome of coronavirus disease 2019 (COVID-19) infection. Diabetes Metab Syndr Clin Res Rev 2020;14:16135.

17. Scheen AJ. Statins and clinical outcomes with COVID-19: Meta-analyses of observational studies. Diabetes Metab 2020;47:101220.

18. Bikdeli B, Talasaz AH, Rashidi F, et al. Intermediate versus standard-dose prophylactic anticoagulation and statin therapy versus placebo in critically-ill patients with COVID-19: Rationale and design of the INSPIRATION/INSPIRATION-S studies. Thromb Res 2020;196:382-94.
19. Cesaro A, Bianconi V, Gragnano F, et al. Beyond cholesterol metabolism: The pleiotropic effects of proprotein convertase subtilisin/kexin type 9 (PCSK9). Genetics, mutations, expression, and perspective for long-term inhibition. Biofactors 2020;46:367-80.

20. Iqbal Z, Ho JH, Adam S, et al. Managing hyperlipidaemia in patients with COVID-19 and during its pandemic: An expert panel position statement from HEART UK. Atherosclerosis 2020;313:126-36.

21. Mureddu GF, Ambrosetti M, Venturini E, et al. Cardiac rehabilitation activities during the COVID-19 pandemic in Italy. Position Paper of the AICPR (Italian Association of Clinical Cardiology, Prevention and Rehabilitation). Monaldi Arch Chest Dis 2020;90:1439.

22. Gragnano F, Natale F, Concilio C, et al. Adherence to proprotein convertase subtilisin/kexin 9 inhibitors in high cardiovascular risk patients: An Italian single-center experience. J Cardiovasc Med 2018;19:75-7.

23. Cesaro A, Gragnano F, Fimiani F, et al. Impact of PCSK9 inhibitors on the quality of life of patients at high cardiovascular risk. Eur J Prev Cardiol 2020;27:556-8.

24. Kotseva K, De Backer G, De Bacquer D, et al. Lifestyle and impact on cardiovascular risk factor control in coronary patients across 27 countries: Results from the European Society of Cardiology ESC-EORP EUROASPIRE V registry. Eur J Prev Cardiol 2019;26:824-35. 